\title{
EFFECTIVENESS OF VISUALISATIONS FOR DETECTION OF ERRORS IN SEGMENTATION OF BLOOD VESSELS
}

\author{
B.W. van Schooten, E.M.A.G. van Dijk \\ Human Media Interaction, University of Twente, Enschede, Netherlands \\ schooten@ewi.utwente.nl,bvdijk@ewi.utwente.nl \\ A. Suinesiaputra, J.H.C. Reiber \\ LKEB, Leiden University Medical Center, Leiden, Netherlands \\ A.Suinesiaputra@lumc.nl, J.H.C.Reiber@lumc.nl
}

Keywords: volume visualization, segmentation, radiology, MRA, visual cues.

Abstract: Vascular disease diagnosis often requires a precise segmentation of the vessel lumen. When 3D (Magnetic Resonance Angiography, MRA, or Computed Tomography Angiography, CTA) imaging is available, this can be done automatically, but occasional errors are inevitable. So, the segmentation has to be checked by clinicians. This requires appropriate visualisation techniques. A number of visualisation techniques exist, but there has been little in the way of user studies that compare the different alternatives. In this study we examine how users interact with several basic visualisations, when performing a visual search task, checking vascular segmentation correctness of segmented MRA data. These visualisations are: direct volume rendering (DVR), isosurface rendering, and curved planar reformatting (CPR). Additionally, we examine if visual highlighting of potential errors can help the user find errors, so a fourth visualisation we examine is DVR with visual highlighting. Our main findings are that CPR performs fastest but has higher error rate, and there are no significant differences between the other three visualisations. We did find that visual highlighting actually has slower performance in early trials, suggesting that users learned to ignore them.

\section{INTRODUCTION}

Vascular disease diagnoses can be done effectively by means of 3D imaging techniques such as magnetic resonance angiography (MRA) and computed tomography angiography (CTA). We focus on contrastenhanced MRA as this is where our current expertise lies (Suinesiaputra et al., 2009). With help of these imaging techniques, the thickness of the inside of the vessel (the vessel lumen) can be segmented precisely, and assessed quantitatively. However, manual segmentation is time-consuming. Segmentation can also be done automatically, but occasional errors are currently inevitable. So we argue that, for both manual and automatic segmentation, it is important to visualise both segmentation and the raw data using appropriate visualisation techniques, so that both can be examined and compared at will.

A number of visualisations have been developed to help interpret the 3D image data and the segmentation (see (van Schooten et al., 2009) for an overview). However, there exists little in the way of clinical or user studies that compare different visualisations.

We conducted an experiment where we compare user performance (time taken and error rate) w.r.t. different visualisations, by having users find errors in a vascular-like structure with automatic segmentation added. Navigation (translation and rotation) is required to view the entire vessel. The four most promising visualisations we consider are direct volume rendering (DVR) (Mueller et al., 2005) versus several alternatives that provide extra cues for detecting errors: curved planar reformation (CPR) (Kanitsar, 2004), isosurface, and explicit highlighting of suspicious areas in the DVR in a different colour.

Our segmentation consists of a pathline and a triangle mesh indicating the lumen. The pathline is a line that goes through the lumen of the vessel, which is determined as a first step of the automatic segmentation process (Boskamp et al., 2004). The pathline is also used for determining the curved plane in CPR. Accuracy of current algorithms is high enough so that the pathline is accurate in most areas, and is at least close to the actual vessel in areas where it is inaccu- 
rate. This is essential for using the CPR for examining the segmentation.

The contrast-enhanced MRA imaging technique detects a previously administered intravenous contrast agent. One advantage is that high densities almost always represent blood vessels rather than other features of the human body, so little pre-filtering is required to remove distractors and leave only the vessels. A disadvantage is that density is not uniform across the image: it may be lower in places where blood flows less quickly. In some cases where blood flow is blocked by a stenosis, a length of vessel may even be blacked out. These features make it harder for algorithms to segment the vessels.

It is our experience that segmentation errors are usually close to a visually apparent local density decreases in the MRA. This indicates the most common types of segmentation error: crossing through vessel boundaries (in cases of high tortuosity or stenosis), and thickness determination problems near bifurcations. See fig. 1 for examples. If we assume there is always a local density decrease, we can more easily assess the different visualisations. We will call this the density deviation assumption. If we do not make this assumption, our assessment depends on the particular segmentation algorithm.

In order to reason about visualisations in the light of our error detection task, we define an error detection cue as a clear visual cue that shows that there is a potential error in the segmentation. The cue may be explicit, showing potential errors by explicit visual highlighting such as a different colour, or implicit, showing them by an easily interpreted visual configuration. Any such cue can be argued to have a particular level of sensitivity (what percentage of errors are detected) and specificity (how many false detections are generated).

Perceptual studies on explicit error detection cues have been made in several domains, including generic perceptual stimuli, and perception in medical and security applications. (Fisher and Tan, 1989) and (Tamborello and Byrne, 2007) found that, for higher highlighting accuracies, response time progressively decreased for the accurate highlighting cases, but it also progressively increased for the inaccurate cases. The result is theoretically non-optimal, and in their study, error detection cues had a detrimental effect. Other studies found that for the presence of false alarms, users double-check the data (Dixon et al., 2007), resulting in slower performance, or overrely on the cues, resulting in higher error rate (Maltz and Shinar, 2003). Additionally, distinction of visual elements by highlighting helps focussed attention but hinders integrative attention (that is, when all visual stimuli need

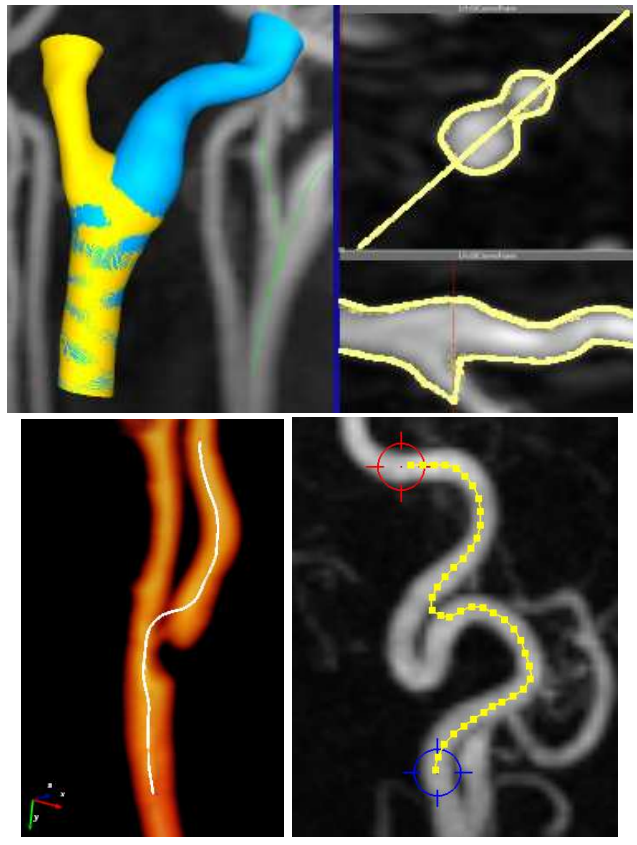

Figure 1: Density deviation around error areas in real life data. Top: segmentation error in bifurcation. Top left: a bifurcating vessel with separate segmentations for each bifurcation. Top right: cross-sections of the bifurcating vessel, showing that the blue (right side) segmentation follows partially down the wrong vessel. Bottom left: the pathline bypasses a stenotic area in the right bifurcation. Bottom right: segmentation error in tortuous area. The pathline skips part of the vessel's curvature.

to be integrated) (Wickens and Andre, 1990). Another detrimental effect is called attention tunneling, which means the highlights distract the user from seeing other elements in the scene (Yeh and Wickens, 2001).

We shall now focus on the four visualisations that we shall evaluate. See fig. 2.

- DVR is a commonly used visualisation. We visualise the segmentation in DVR by means of a wireframe mesh. DVR will show most segmentation errors clearly, but only when the model is rotated suitably. The error detection cues are implicit.

- CPR is a curved cross-section, following a vessel's pathline. This results in useful crosssectional information along the entire vessel, capable of removing distracting parts of the volume data (Achenbach et al., 1998). Because our vessels curve in all directions, we use the straightened variant of CPR, where the cross-section is topologically distorted so that the pathline becomes a straight line. The intersection of the segmenta- 
tion with the curved plane is represented as a line. This enables the user to see immediately where the segmentation crosses a lower-density area, in most cases without any rotation. The visual cue is simply the line not following the densest parts of the vessel. This is an implicit error detection cue.

- Isosurface has strong error detection cues for a properly chosen density threshold and raw data with relatively uniform density. Then, the segmentation goes outside of the surface boundary where an error occurs. This can be explicitly visualised using occlusion cues, though some camera rotation may be required to see them. This capability has $100 \%$ specificity under the density deviation assumption, but less than $100 \%$ sensitivity because areas where the segmentation is thinner than required are not marked.

A disadvantage is that for realistic data with nonuniform density, a plain isosurface often will not be representative for the vessel lumen. Nevertheless, we argue that the potential benefits of isosurface-type approaches are worth studying.

- Suspicious area highlighting. Here, an algorithm marks the suspicious areas, using knowledge of typical segmentation errors. In some cases, the segmentation algorithm itself outputs a credibility level for every part of its output, in which case suspicious area information will be easy to obtain. In model based algorithms for example, low conformance to the model naturally signals a suspicious area (Levy et al., 2007). The areas can be shown as visual highlights in almost any visualisation. We show them in DVR in our study.

Suspicious area highlighting can be considered experimental, but has been most actively researched in colonoscopy and mammography. One clinical study on mammography found it useful (Freer and Ulissey, 2001). Here, they help the clinician find the locations of resp. polyps and masses/microcalcifications. An algorithm parameter $\alpha$ can trade off sensitivity for specificity. In the areas of colonoscopy and mammography, some of these algorithms manage to reach near $100 \%$ sensitivity (i.e. no false negatives) while minimising false positives to an acceptable level (i.e. not needing to mark a substantial part of the image) (Hong et al., 2006; López-Aligué et al., 2004). Others only manage $85 \%-90 \%$ sensitivity (Wang et al., 2007) or less.

There has been more limited research in the area of segmentation error detection. (Levy et al., 2007) proposes an error detection algorithm for
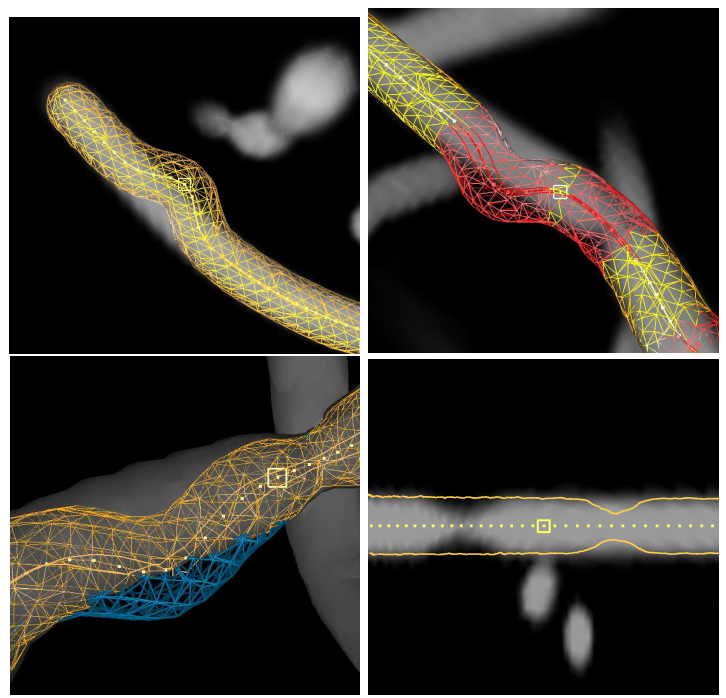

Figure 2: The visualisations used in the experiment. The pictures visualise software phantoms with two types of artificial segmentation errors: veering errors (segmentation veers from vessel) and thickness errors (segmentation and vessel thickness do not match). Top left: DVR with veering error in the center. Top right: DVR with suspicious area highlighting in red; highlighted veering error at top left, highlighted thickness error at bottom right. Bottom left: isosurface with veering error in the middle; the segmentation is marked in blue where it sticks out. Bottom right: CPR with thickness errors at the left and right.

Table 1: The four visualisations.

$\begin{array}{lllll}\text { Name } & \text { type } & \text { sensit. } & \text { specif. } & \begin{array}{l}\text { requires } \\ \text { rotation }\end{array} \\ \text { DVR } & \text { implicit } & 100 \% & 100 \% & \text { yes } \\ \text { CPR } & \text { implicit } & 100 \% & 100 \% & \text { rarely } \\ \text { Isosurf. } & \text { explicit } & <100 \% & 100 \% & \text { yes } \\ \text { Susp.Ar. } & \text { explicit } & \text { depends } & \text { depends } & \text { no }\end{array}$

bladder segmentation. It can reach $100 \%$ sensitivity only by raising false positives to an unacceptable level (i.e. by marking a substantial part of the image). (Levinski et al., 2009) also marks suspicious areas in their brain segmentation system. They claim an efficiency improvement of a factor 5 for their overall segmentation system, but the benefits of the suspicious area highlighting system by itself are unclear. Both proposals make use of knowledge of or internal parameters within the segmentation algorithm. 

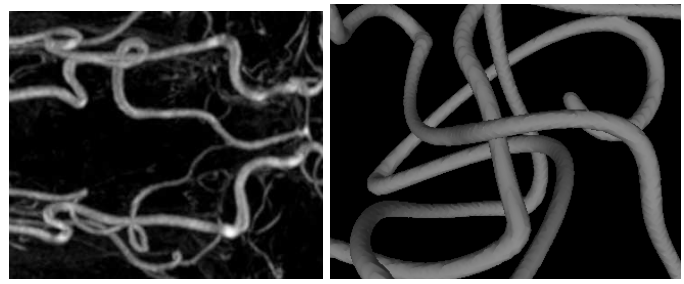

Figure 3: Left: real life data (MRA of carotid arteries). Right: typical software phantom.

\section{EXPERIMENTAL DESIGN}

\subsection{Task}

In our study, users had to perform a visual search task that consists of checking the correctness of automatic segmentations of vessels in MRA scans.

The MRA scans are artificially generated (also known as software phantoms), along with segmentations with artificially generated segmentation errors. This way it is easy to generate dozens of cases with a clear distinction between correct and erroneous, an unambiguous ground truth, and similar difficulty levels. We generated these software phantoms in a way similar to previous work (van Schooten et al., 2009). See also (Rolland et al., 1995) for a similar approach.

A vessel is constructed using a sum of sine waves. Three distractors vessels were added in each phantom. Thickness of the vessel was varied in a stylized manner with thinner and thicker areas. When looking at a cross-section, density in the center of the vessel was highest, gradually lowering towards the boundaries of the vessel, and zero outside of the vessel. No noise or other distractors were added, neither were bifurcations present. See figure 3.

Errors are defined as a deviation between the segmentation and the densest parts of the volume. Only three error types exist: a veering away of the pathline and segmentation from the vessel, the segmentation being thinner than the vessel, and the segmentation being thicker. These are explained to the user as part of an interactive tutorial. See figure 2.

We generated 6-8 segmentation errors per model, with approximately equal numbers of veering and thickness errors. The number of errors was varied to ensure the search was not self-terminating. Additionally, we generated suspicious areas for the visual highlighting condition. Both false positives and negatives were generated: between 1 and 3 of each in each trial, yielding an average of $25 \%$ false positive and negatives.

\subsection{Controls}

Because of the limited amount of previous research, our choice of navigation controls is partially exploratory. We chose controls to be as simple as possible without sacrificing user control. We did want users to be able to rotate and zoom at will. Control is with the mouse only, and each button is designed to map to a single function as much as possible. We have two essentially different navigation methods, namely CPR (essentially 2D, plus rotation around the vessel axis), and the $3 \mathrm{D}$ visualisations.

One major choice we made is to base navigation on the pathline. The camera is always centered around a point on the pathline. This greatly reduces the number of degrees of freedom required for $3 \mathrm{D}$ navigation and makes it much easier to do with a regular (2D) mouse. This does assume that the pathline, if not accurate, is at least in the vicinity of the target vessel, which we found to be true in our experience. The user can navigate along the pathline by either using the mouse wheel or by clicking on a pathline point with the middle mouse button (MMB). In 3D, the camera is also rotated so that the relative angle of the vessel w.r.t. the viewer remains the same.

In $3 \mathrm{D}$, the model is rotated by moving the mouse while the right mouse button (RMB) is held. A standard "two-axis valuator" method is used (Bade et al., 2005). A fourth button below the left button (the left side-button or LSMB) was used to zoom and rotate around the view axis, by resp. moving the mouse vertically and horizontally with the button pressed. In $\mathrm{CPR}$, there is only one rotational degree of freedom. Both the RMB + moving vertical, or the LSMB + moving horizontal, was used to rotate around the vessel axis.

The camera starts in the middle of the vessel and is zoomed out to provide an overview. We chose to do this so users do not get disoriented. We did ask users to proceed systematically, that is, from end to end.

The selection of errors is designed to take as little effort as possible; the user's task concentrates only on finding the errors. When the user clicks with the left button (LMB-this is a traditional choice that many users like) on a part of the pathline that contains an error, the error is selected.

\subsection{Setup}

We used an within-subjects design for the four conditions, which we name: DVR, DVRSUS (DVR with suspicious areas highlighted in red), ISOSURF (Isosurface with non-occluded segmentation shown in blue), and CPR. The pathline was visualised in yel- 
low, the segmentation either as an orange mesh (in 3D) or as an orange line intersecting the CPR plane (in CPR). Each condition was repeated four times. Trials took about 25-80 seconds. All users received the same set of software phantoms in the same order, but with different, randomly ordered and counterbalanced, conditions. The users had to complete 4 trials per condition, totaling 16 trials. This was achieved by cycling through the conditions 4 times, each time in a different order, effectively counterbalancing for learning effects within a single session. Total time for the entire session was about 30-40 minutes. At the end of a trial, time taken and number of errors made was recorded, and shown to the user, before the next trial began. The experiment was concluded with a subjective survey. See table 2.

The subjects were seated in a comfortable chair about $70 \mathrm{~cm}$ away from a 24" computer screen. An experimenter was seated behind them. The controls and task were explained and trained in a 12-15 minute interactive tutorial.

\subsection{Participants}

Because we used somewhat stylised models, medical laypersons could easily do the task. Since our research concerns usability involving novel interaction techniques, we asked experts on user interfaces rather than medical experts to perform our experiment, so as to reduce learning difficulties and get better feedback. We recruited 12 participants from the Human Media Interaction department of our CS faculty, invited through personal invitation.

The users' ages ranged from 25 to 51 years, with an average of 32, and 2 users above 40. Four users were female. Most were Ph.D. students, but there was 1 student and 3 who finished their Ph.D. All users used the mouse in their right hand.

As regards experience, all were highly experienced computer users, and about half also had experience with actual development of user interfaces. None had experience with medical 3D scans, except one user who claimed occasional experience. Most users had occasional 3D CAD/modelling experience except 2 who had intensive experience and 3 who had none.

\section{RESULTS}

\subsection{Main Results}

Overall, there were no real problems with interaction, and the task was successfully performed by all users.
Users quickly learned to identify errors with help of the tutorial. Some users kept making mistakes in the controls during the main trial (pressing the wrong buttons), but none of these mistakes were costly in terms of ruining the task performance. Most mistakes could be corrected in a fraction of a second. The error rate (number of errors missed and falsely identified errors) was near zero for almost all users. Users found the graphical render quality good, with all users finding the animation smooth enough, and only 3 users finding some details somewhat hard to see. See table 3.

We expect time performance data to be multiplicative rather than additive, so to analyse time performance, we transformed the data using the log transform. We used a second transformation to increase sensitivity. It is based on the fact that the sequence of trials was the same for all users. We divided the time for each trial by the overall average of that trial over all users (note that all conditions occurred equally often). This has the effect of normalising for variations in trial difficulty.

We then performed a repeated-measures ANOVA with a Sidak post-hoc analysis. $(F(3,33)=$ $16.384, p<=0.0005)$. We found that CPR was by far the fastest $(p=0.008)$. This seems to be attributable to the fact that users could see all errors without needing to rotate. However, differences between the other visualisations were not significant. The averages were also very close to each other, see the following table.

$\begin{array}{lllll}\text { cond. } & \text { CPR } & \text { DVR } & \text { DVRSUS } & \text { ISOSURF } \\ \text { perf.(s) } & 43.28 & 60.44 & 68.00 & 64.96\end{array}$

Because of the difficulty of the task, we may expect learning effects to occur during the experiment. If we look at the progress over time, it seems that the conditions go through different learning curves. DVRSUS and ISOSURF seem slower at first, then become equally fast as DVR.

We analysed the learning effect by grouping the trials into four phases. Recall that one session consists of four cycles, each cycling through all four conditions. We analysed each cycle separately, as well as cycle 1 and 2 together (the first eight trials) and cycle 3 and 4 together (the last eight trials). The average time performance over all users for each of these cycles is plotted in figure 4. Different learning effects are apparent, especially in cycle 1 . For each cycle, we used again a repeated-measures Anova with Sidak post-hoc analysis, considering all conditions except CPR. We found that DVR was faster than DVRSUS in the first eight trials ( $p=0.015)$, and also that DVR was faster than both DVRSUS and ISOSURF in the first four trials $(p<=0.036)$. No other significant differences were found.

While these results are tentative, we can conclude 
Table 2: List of all mean time performance variables and all subjective variables, and the scales used.

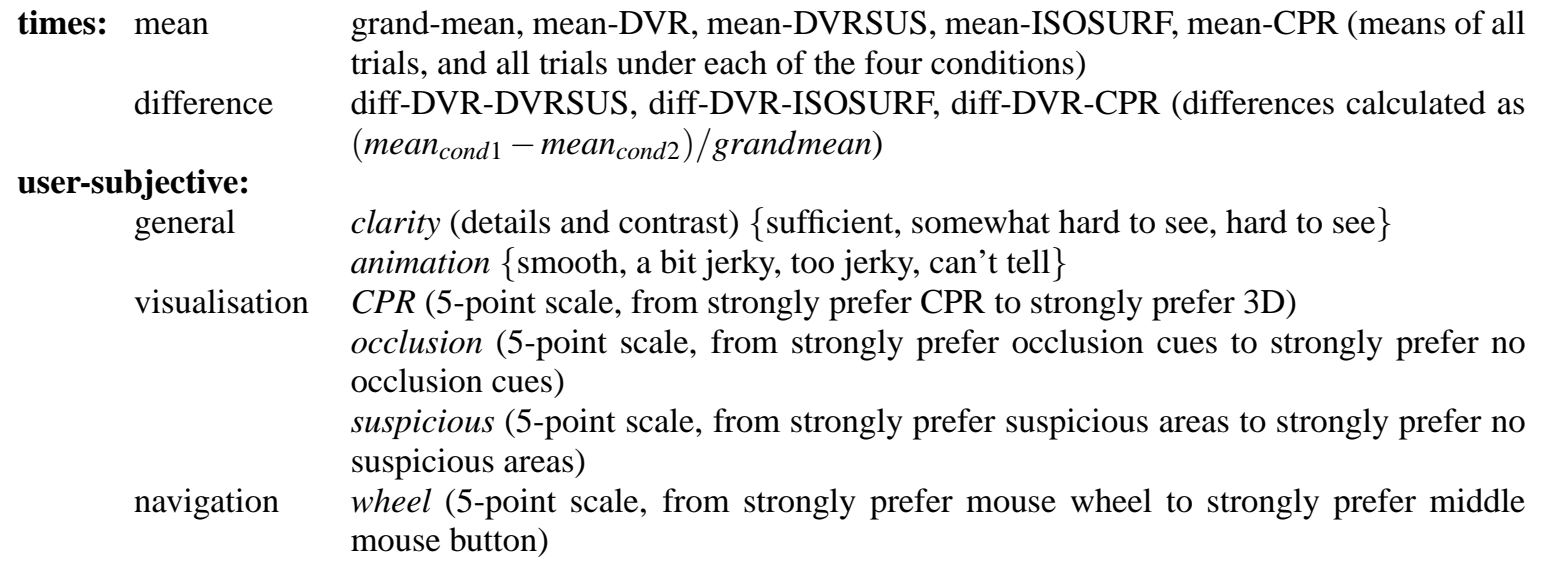

that DVRSUS and ISOSURF have a steeper learning curve, possibly caused by users learning to process the cues, or ignore them. This is consistent with our qualitative observations, which indicate that users spent a noticeable amount of time double-checking the suspicious areas, which gradually became less. One user even remarked: you quickly learn to ignore the suspicious areas. At the end of the experiment however, DVRSUS and ISOSURF fail to provide significant improvement, although there may be a small improvement which can only be measured by a more sensitive experiment.

Mistakes (user errors) were made in $17 \%$ of the trials, most of which were single mistakes. If we consider the number of mistakes per clickable vessel region, only $3.4 \%$ of the user selections were erroneous, almost all of which were omitted regions. We analysed mistakes by means of a $\chi^{2}$ table, assuming that trials and selection of selectable regions are independent events.

$\begin{array}{lllll}\text { condition } & \text { trials } & \begin{array}{c}\text { segm. } \\ \text { errors }\end{array} & \begin{array}{c}\text { mistakes trials } \\ \text { w/ mis- } \\ \text { takes }\end{array} \\ \text { CPR } & 48 & 336 & 19 & 16 \\ \text { DVR } & 48 & 336 & 12 & 7 \\ \text { DVRSUS } & 48 & 336 & 10 & 7 \\ \text { ISOSURF } & 48 & 336 & 5 & 3\end{array}$

A $\chi^{2}$ goodness of fit test over trails with and without mistakes reveals $\chi^{2}(3, N=192)=13.3, p=$ 0.0041 , a $\chi^{2}$ test over total segmentation errors and total number of user mistakes reveals a lower value, $\chi^{2}(3, N=1344)=9.094, p=0.028$, but still significant. Apparently, users made more mistakes in CPR and possibly less in ISOSURF. Contrary to theory, we did not find that users made more mistakes in the explicit-cues conditions DVRSUS and ISOSURF.

We will now consider subjective preferences. See
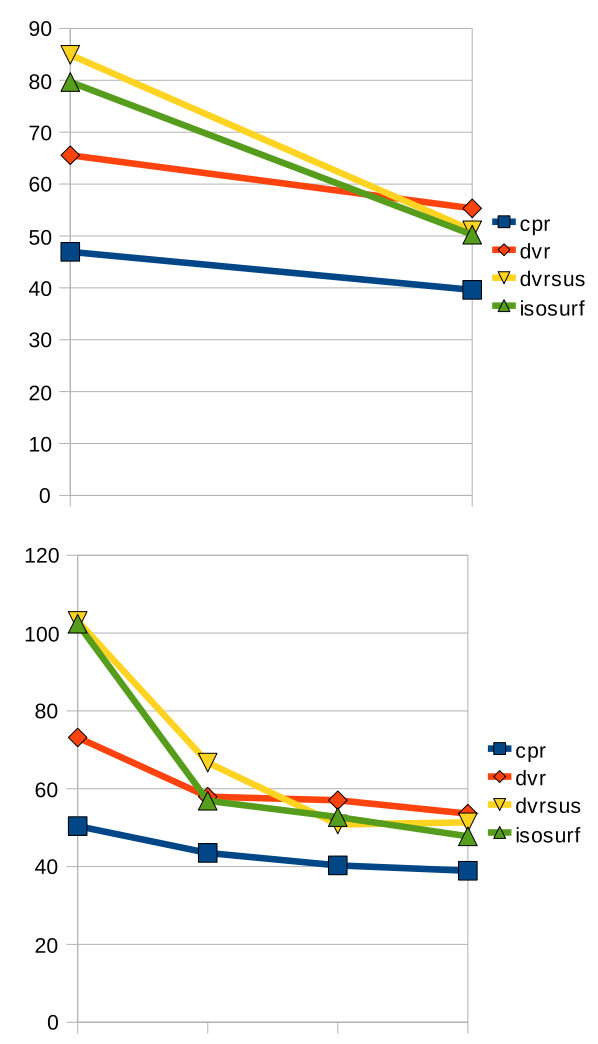

Figure 4: Performance improvement over time. The Y axis denotes average trial performance in seconds. Top: first 8 trials versus last 8 trials. Bottom: from left to right: trials $1-4,5-8,9-12$, and 13-16.

table 3. Users' preferences for the different conditions were not always consistent with their performance. Users were strongly divided about CPR, either preferring or dispreferring it strongly, with an av- 
Table 3: Subjective variable statistics. Number of users who selected each item on each survey scale, and the average value.

\begin{tabular}{lcccccc} 
variable & \multicolumn{4}{c}{ nr. users: } & average \\
& 1 & 2 & 3 & 4 & 5 & \\
CPR & 5 & 2 & - & 1 & 4 & 2.75 \\
occlusion & - & 1 & 2 & 4 & 5 & 4.08 \\
suspicious & - & 2 & 2 & 4 & 4 & 3.83 \\
\hline wheel & 2 & 1 & - & 1 & 8 & 4.00 \\
clarity & - & 3 & 9 & & & 2.75 \\
animation & - & - & 12 & & & 3.00
\end{tabular}

erage slightly below neutral, even though CPR scores significantly faster on average. We used the nonparametric Kendall's $\tau$ to check if there was a correlation between preference and time performance. We found a significant positive correlation, both absolute (mean-CPR: $p=0.046$ ) and relative (diff-DVR-CPR: $p=0.004)$.

As concerns occlusion cues and suspicious area highlighting, most users preferred having them, and no-one strongly preferred their absence. We again correlated them with time performance using Kendall's $\tau$, but found no significant correlations with either mean-ISOSURF, diff-DVR-ISOSURF, meanDVRSUS, or diff-DVR-DVRSUS.

\subsection{Controls}

In order to find out how the controls were used, we analysed the interaction data, in particular the use of mouse wheel and MMB, and time spent rotating and zooming. Users spent only an average of $0.51 \mathrm{sec}$. per trial on zooming, and $0.24 \mathrm{sec}$. on rotating around the view axis. In contrast, they spent around 11.5 sec. (18.3\% of the time) on rotating around the other two axes using the RMB. Zoom behaviour differed between users. Most ( 8 of 12) users would navigate to one end, and then zoom in quite far to see details clearly, then proceed to the other end without zooming out. So, a majority of users proceeded systematically with a fixed zoom level. Only four users liked to zoom in and out in particular cases to see context better. We conclude that the RMB rotation controls are by far the most important, and that a fixed zoom level would probably suffice for this task.

As regards mouse wheel and MMB, we found that most users almost exclusively used the mouse wheel. The MMB was clicked only 2.13 times per trial on average. However, there were two users who used the MMB almost exclusively. These are the two users who strongly preferred the MMB in the subjective survey. We conclude that the choice between mouse wheel and MMB is a matter of personal choice.

In a few cases, users forgot what was back and forward in their systematic search from one end to the other, and would navigate or even zoom out to check the direction. It may be worth incorporating a feature that ensures users do not get lost this way.

A few users did not proceed quite systematically, and would go back and forth randomly to check things they think might be suspicious, or go outwards from the middle. In only a couple of cases, users changed strategies in the middle of the experiment, which may be considered a learning effect.

\section{CONCLUSIONS}

We conducted an experiment which compared task performance and subjective preference for a visual search task involving finding segmentation errors in segmented MRA data. We compared four visualisations, namely DVR, CPR, Isosurface (ISOSURF), and DVR with suspicious areas highlighted (DVRSUS). We used time taken and error rate as performance measures.

CPR was clearly the fastest, but also has higher error rate. Users were strongly divided over their preference for CPR, reflecting their relative individual time performance differences. We do not have an explanation for the higher error rate. CPR showed all errors in our models without rotation, so it is not caused by the users rotating too little to discover all errors. Possibly the high interaction speed also made users double-check less often. It would be interesting to find out why error rate was higher in a future experiment.

Contrary to some previous studies (e.g. (Maltz and Shinar, 2003)), we found that suspicious area highlighting (as in ISOSURF and DVRSUS) did not lead to increased error rate as compared to DVR. However, no significant time performance improvement is seen for either DVRSUS or ISOSURF over DVR either, suggesting that the error detection cues in these visualisations did not help. Looking at the learning effects over time showed that they performed slower than DVR in early trials, but equally fast in later trials. This is likely because of the double checking involved in the warning cues, and at least some of the users simply learned to ignore the cues. However, most users did prefer both DVRSUS and ISOSURF over DVR.

Possibly, the probability of false positives and negatives in DVRSUS (averaging 25\% each) was too high for this feature to be useful. Also, because of learning effects, our experiment can be made more 
sensitive by increasing the number of trials. So, it is still possible that some users benefited or would benefit from suspicious areas, but this requires a new experiment to find out.

As regards zoom and rotate controls, we found that traditional rotation around the $\mathrm{X} / \mathrm{Y}$ axis was the most important. This two degrees of freedom rotation appeared sufficient to discover all errors in the $3 \mathrm{D}$ visualisations easily. Zooming and rotation around the view $(Z)$ axis were little used. Most users zoomed in to a fixed zoom level and stayed there during the rest of the trial. This suggests that controls can be simplified using a sensible default for zoom level.

\section{ACKNOWLEDGEMENTS}

This work was carried out under the NWO (Netherlands Organization for Scientific research) Multivis project (N 643.100.602), which is part of the NWO VIEW program.

\section{REFERENCES}

Achenbach, S., Moshage, W., Ropers, D., and Bachmann, K. (1998). Curved multiplanar reconstructions for the evaluation of contrast-enhanced electron-beam CT of the coronary arteries. American Journal of Roentgenology, pages 895-899.

Bade, R., Ritter, F., and Preim, B. (2005). Usability comparison of mouse-based interaction techniques for predictable 3D rotation. In 5th international symposium on smart graphics: SG 2005, pages 138-150. Springer.

Boskamp, T., Rinck, D., Link, F., Kümmerlen, B., Stamm, G., and Mildenberger, P. (2004). New vessel analysis tool for morphometric quantification and visualization of vessels in CT and MR imaging data sets. Radiographics, 24(1):287-297.

Dixon, S. R., Wickens, C. D., and McCarley, J. S. (2007). On the independence of compliance and reliance: are automation false alarms worse than misses? Human factors, 49(4):564-72.

Fisher, D. L. and Tan, K. C. (1989). Visual displays: The highlighting paradox. Human Factors, 31(1):17-30.

Freer, T. W. and Ulissey, J. M. (2001). Screening mammography with computer-aided detection: prospective study of 12,860 patients in a community breast center. Radiology, 220:781-786.

Hong, W., Qiu, F., and Kaufman, A. (2006). A pipeline for computer aided polyp detection. IEEE Transactions on Visualization and Computer Graphics, 12(5):861868.
Kanitsar, A. (2004). Curved Planar Reformation for Vessel Visualization. $\mathrm{PhD}$ thesis, Institute of Computer Graphics and Algorithms, Vienna University of Technology, Favoritenstrasse 9-11/186, A-1040 Vienna, Austria.

Levinski, K., Sourin, A., and Zagorodnov, V. (2009). 3D visualization and segmentation of brain MRI data. In GRAPP 2009, pages 111-118.

Levy, J. H., Broadhurst, R. R., Ray, S., Chaney, E. L., and Pizer, S. M. (2007). Signaling local non-credibility in an automatic segmentation pipeline. In Proceedings of the International Society for Optical Engineering meetings on Medical Imaging, Volume 6512.

López-Aligué, F. J., Acevedo-Sotoca, I., García-Manso, A., García-Orellana, C. J., and Gallardo-Caballero, R. (2004). Microcalcifications detection in digital mammograms. In EMBC 2004.

Maltz, M. and Shinar, D. (2003). New alternative methods of analyzing human behavior in cued target acquisition. Human Factors, 45(2):281-295.

Mueller, D. C., Maeder, A. J., and O'Shea, P. J. (2005). Enhancing direct volume visualisation using perceptual properties. In Proc. SPIE, Vol. 5744, pages 446-454.

Rolland, J. P., Muller, K. E., and Helvig, C. S. (1995). Visual search in medical images: a new methodology to quantify saliency. In Proc. SPIE Vol. 2436, pages 4048.

Suinesiaputra, A., de Koning, P. J., Zudilova-Seinstra, E. V., Reiber, J. H. C., and van der Geest, R. J. (2009). A 3D MRA segmentation method based on tubular NURBS model. In International Society for Magnetic Resonance in Medicine 2009, Honolulu, Hawaii.

Tamborello, F. P. and Byrne, M. D. (2007). Adaptive but non-optimal visual search behavior with highlighted displays. Cognitive Systems Research, 8(3):182-191.

van Schooten, B. W., van Dijk, E. M. A. G., ZudilovaSeinstra, E. V., de Koning, P. J. H., and Reiber, J. H. C. (2009). Evaluating visualisation and navigation techniques for interpretation of MRA data. In GRAPP 2009, pages 405-408.

Wang, Y., Gao, X., and Li, J. (2007). A feature analysis approach to mass detection in mammography based on RF-SVM. In ICIP 07, pages 9-12.

Wickens, C. D. and Andre, A. D. (1990). Proximity compatibility and information display: Effects of color, space, and objectness on information integration. $\mathrm{Hu}$ man Factors, 32(1):61-77.

Yeh, M. and Wickens, C. D. (2001). Display signaling in augmented reality: Effects of cue reliability and image realism on attention allocation and trust calibration. Human Factors, 43(3):355-365. 